\title{
Improving the Performance of maxRPC
}

\author{
Thanasis Balafoutis ${ }^{1}$, Anastasia Paparrizou ${ }^{2}$, Kostas Stergiou $^{1,2, \star}$, and Toby Walsh ${ }^{3}$ \\ 1 Department of Information and Communication Systems Engineering, \\ University of the Aegean, Greece \\ 2 Department of Informatics and Telecommunications Engineering, \\ University of Western Macedonia, Greece \\ 3 NICTA, University of New South Wales, Australia
}

\begin{abstract}
Max Restricted Path Consistency (maxRPC) is a local consistency for binary constraints that can achieve considerably stronger pruning than arc consistency. However, existing maxRPC algorithms suffer from overheads and redundancies as they can repeatedly perform many constraint checks without triggering any value deletions. In this paper we propose techniques that can boost the performance of maxRPC algorithms. These include the combined use of two data structures to avoid many redundant constraint checks, and heuristics for the efficient ordering and execution of certain operations. Based on these, we propose two closely related maxRPC algorithms. The first one has optimal $\mathrm{O}\left(e n d^{3}\right)$ time complexity, displays good performance when used stand-alone, but is expensive to apply during search. The second one has $\mathrm{O}\left(e \mathrm{n}^{2} \mathrm{~d}^{4}\right)$ time complexity, but a restricted version with $\mathrm{O}\left(e n d^{4}\right)$ complexity can be very efficient when used during search. Both algorithms have $\mathrm{O}(e d)$ space complexity when used stand-alone. However, the first algorithm has $\mathrm{O}(e n d)$ space complexity when used during search, while the second retains the $\mathrm{O}(e d)$ complexity. Experimental results demonstrate that the resulting methods constantly outperform previous algorithms for maxRPC, often by large margins, and constitute a more than viable alternative to arc consistency.
\end{abstract}

\section{Introduction}

maxRPC is a strong domain filtering consistency for binary constraints introduced in 1997 by Debruyne and Bessiere [5]. maxRPC achieves a stronger level of local consistency than arc consistency (AC), and in [6] it was identified, along with singleton AC (SAC), as a promising alternative to AC. Although SAC has received considerable attention since, maxRPC has been comparatively overlooked. The basic idea of maxRPC is to delete any value $a$ of a variable $x$ that has no arc consistency (AC) or path consistency (PC) support in a variable $y$. A value $b$ is an AC support for $a$ if the two values are compatible, and it is also a PC support for $a$ if this pair of values is path consistent. A pair of values $(a, b)$ is path consistent iff for every third variable there exists at least one value, called a PC witness, that is compatible with both $a$ and $b$.

The first algorithm for maxRPC was proposed in [5], and two more algorithms have been proposed since then [7[10]. The algorithms of [5] and [10] have been evaluated on

\footnotetext{
* Part of this work was carried out when the 3rd author was at NICTA, Australia.
} 
random problems only, while the algorithm of [7] has not been experimentally evaluated at all. Despite achieving considerable pruning, existing maxRRC algorithms suffer from overhead and redundancies as they can repeatedly perform many constraint checks without triggering any value deletions. These constraint checks occur when a maxRPC algorithm searches for an AC support for a value and when, having located one, it checks if it is also a PC support by looking for PC witnesses in other variables. As a result, the use of maxRRC during search often slows down the search process considerably compared to AC, despite the savings in search tree size.

In this paper we propose techniques to improve the applicability of maxRPC by eliminating some of these redundancies while keeping a low space complexity. We also investigate approximations of maxRPC that only make slightly fewer value deletions in practice, while being significantly faster. We first demonstrate that we can avoid many redundant constraint checks and speed up the search for AC and PC supports through the careful and combined application of two data structures already used by maxRPC and AC algorithms [7]10|2[8|9]. Based on this, we propose a coarse-grained maxRPC algorithm called maxRPC 3 with optimal $\mathrm{O}\left(e n d^{3}\right)$ time complexity. This algorithm displays good performance when used stand-alone (e.g. for preprocessing), but is expensive to apply during search. We then propose another maxRPC algorithm, called maxRPC $3^{r m}$. This algorithm has $\mathrm{O}\left(e n^{2} d^{4}\right)$ time complexity, but a restricted version with $\mathrm{O}\left(e n d^{4}\right)$ complexity can be very efficient when used during search through the use of residues. Both algorithms have $\mathrm{O}(e d)$ space complexity when used stand-alone. However, maxRPC 3 has $\mathrm{O}($ end $)$ space complexity when used during search, while maxRPC $3^{r m}$ retains the $\mathrm{O}(e d)$ complexity.

Similar algorithmic improvements can be applied to light maxRPC (lmaxRPC), an approximation of maxRPC [10]. This achieves a lesser level of consistency compared to maxRPC but still stronger than AC, and is more cost-effective than maxRPC when used during search. Experiments confirm that lmaxRPC is indeed a considerably better option than maxRPC.

We also propose a number of heuristics that can be used to efficiently order the searches for PC supports and witnesses. Interestingly, some of the proposed heuristics not only reduce the number of constraint checks but also the number of visited nodes.

We make a detailed experimental evaluation of new and existing algorithms on various problem classes. This is the first wide experimental study of algorithms for maxRPC and its approximations on benchmark non-random problems. Results show that our methods constantly outperform existing algorithms, often by large margins. When applied during search our best method offers up to one order of magnitude reduction in constraint checks, while cpu times are improved up to four times compared to the best existing algorithm. In addition, these speed-ups enable a search algorithm that applies lmaxRPC to compete with or outperform MAC on many problems.

\section{Background and Related Work}

A Constraint Satisfaction Problem (CSP) is defined as a tuple $(X, D, C)$ where: $X=$ $\left\{x_{1}, \ldots, x_{n}\right\}$ is a set of $n$ variables, $D=\left\{D\left(x_{1}\right), \ldots, D\left(x_{n}\right)\right\}$ is a set of domains, one for each variable, with maximum cardinality $d$, and $C=\left\{c_{1}, \ldots, c_{e}\right\}$ is a set of $e$ 
constraints. Each constraint $c$ is a pair $(\operatorname{var}(c), \operatorname{rel}(c))$, where $\operatorname{var}(c)=\left\{x_{1}, \ldots, x_{m}\right\}$ is an ordered subset of $X$, and $\operatorname{rel}(c)$ is a subset of the Cartesian product $D\left(x_{1}\right) \times \ldots \times$ $D\left(x_{m}\right)$ that specifies the allowed combinations of values for the variables in $\operatorname{var}(c)$. In the following, a binary constraint $c$ with $\operatorname{var}(c)=\left\{x_{i}, x_{j}\right\}$ will be denoted by $c_{i j}$, and $D\left(x_{i}\right)$ will denote the current domain of variable $x_{i}$. Each tuple $\tau \in \operatorname{rel}(c)$ is an ordered list of values $\left(a_{1}, \ldots, a_{m}\right)$ such that $a_{j} \in D\left(x_{j}\right), j=1, \ldots, m$. A tuple $\tau \in \operatorname{rel}\left(c_{i}\right)$ is valid iff none of the values in the tuple has been removed from the domain of the corresponding variable.

The process which verifies whether a given tuple is allowed by a constraint $c$ is called a constraint check. A binary CSP is a CSP where each constraint involves at most two variables. We assume that binary constraint checks are performed in constant time. In a binary CSP, a value $a_{i} \in D\left(x_{i}\right)$ is arc consistent (AC) iff for every constraint $c_{i j}$ there exists a value $a_{j} \in D\left(x_{j}\right)$ s.t. the pair of values $\left(a_{i}, a_{j}\right)$ satisfies $c_{i j}$. In this case $a_{j}$ is called an AC-support of $a_{i}$. A variable is AC iff all its values are AC. A problem is AC iff there is no empty domain in $D$ and all the variables in $X$ are AC.

\section{1 maxRPC}

A value $a_{i} \in D\left(x_{i}\right)$ is max restricted path consistent (maxRPC) iff it is AC and for each constraint $c_{i j}$ there exists a value $a_{j} \in D\left(x_{j}\right)$ that is an AC-support of $a_{i}$ s.t. the pair of values $\left(a_{i}, a_{j}\right)$ is path consistent $(\mathrm{PC})$ [5]. A pair of values $\left(a_{i}, a_{j}\right)$ is PC iff for any third variable $x_{k}$ there exists a value $a_{k} \in D\left(x_{k}\right)$ s.t. $a_{k}$ is an AC-support of both $a_{i}$ and $a_{j}$. In this case $a_{j}$ is a PC-support of $a_{i}$ in $x_{j}$ and $a_{k}$ is a PC-witness for the pair $\left(a_{i}, a_{j}\right)$ in $x_{k}$. A variable is maxRPC iff all its values are maxRPC. A problem is maxRPC iff there is no empty domain and all variables are maxRPC.

To our knowledge, three algorithms for achieving maxRPC have been proposed in the literature so far. The first one, called maxRPC1, is a fine-grained algorithm based on AC6 and has optimal $\mathrm{O}\left(e n d^{3}\right)$ time complexity and $\mathrm{O}(e n d)$ space complexity [5]. The second algorithm, called maxRPC 2 , is a coarse-grained algorithm having $\mathrm{O}\left(e n d^{3}\right)$ time and $\mathrm{O}(e d)$ space complexity [7]. Finally, maxRPC ${ }^{r m}$ is a coarse-grained algorithm based on $\mathrm{AC} 3^{r m}$ [10]. The time and space complexities of maxRPC ${ }^{r m}$ are $\mathrm{O}\left(e n^{2} d^{4}\right)$ and $\mathrm{O}(e n d)$ but it has some advantages compared to the other two because of its lighter use of data structures. Among the three algorithms maxRPC2 seems to be the most promising for stand-alone use as it has a better time and space complexity than maxRPC ${ }^{r m}$ without requiring heavy data structures or complex implementation as maxRPC1 does. On the other hand, maxRPC ${ }^{r m}$ can be better suited for use during search as it avoids the costly maintainance of data structures.

Central to maxRPC2 is the LastPC data structure, as we call it here. For each constraint $c_{i j}$ and each value $a_{i} \in D\left(x_{i}\right)$, LastPC $C_{x_{i}, a_{i}, x_{j}}$ gives the most recently discovered PC-support of $a_{i}$ in $D\left(x_{j}\right)$. maxRPC2 maintains this data structure incrementally. This means that the data structure is copied when moving forward during search (i.e. after a successfully propagated variable assignment) and restored when backtracking (after a failed variable assignment). This results in the following behavior: When looking for a PC-support for $a_{i}$ in $D\left(x_{j}\right)$, maxRPC2 first checks if $\operatorname{LastP} C_{x_{i}, a_{i}, x_{j}}$ is valid. If it is not, it searches for a new PC-support starting from the value immediately after LastPC $C_{x_{i}, a_{i}, x_{j}}$ in $D\left(x_{j}\right)$. In this way a good time complexity bound is achieved. On 
the other hand, maxRPC ${ }^{r m}$ uses a data structure similar to LastPC to store residues, i.e. supports that have been discovered during execution and stored for future use, but does not maintain this structure incrementally]. When looking for a PC-support for $a_{i}$ in $D\left(x_{j}\right)$, if the residue LastPC $C_{x_{i}, a_{i}, x_{j}}$ is not valid then maxRPC ${ }^{r m}$ searches for a new PC-support from scratch in $D\left(x_{j}\right)$. This results in higher complexity, but crucially does not require costly maintainance of the LastPC data structure during search.

A major overhead of both maxRPC2 and maxRPC ${ }^{r m}$ is the following. When searching for a PC-witness for a pair of values $\left(a_{i}, a_{j}\right)$ in a third variable $x_{k}$, they always start the search from scratch, i.e. from the first available value in $D\left(x_{k}\right)$. As these searches can be repeated many times during search, there can be many redundant constraint checks. In contrast, maxRPC1 manages to avoid searching from scratch through the use of an additional data structure. This saves many constraint checks, albeit resulting in $\mathrm{O}($ end $)$ space complexity and requiring costly maintainance of this data structure during search. The algorithms we describe below largely eliminate these redundant constraint checks with lower space complexity, and in the case of maxRPC $3^{r m}$ with only light use of data structures.

\section{New Algorithms for maxRPC}

We first recall the basic ideas of algorithms maxRPC2 and maxRPC ${ }^{r m}$ as described in [7] and [10]. Both algorithms use a propagation list $L$ where variables whose domain is pruned are added. Once a variable $x_{j}$ is removed from $L$ all neighboring variables are revised to delete any values that are no longer maxRPC. For any value $a_{i}$ of such a variable $x_{i}$ there are two possible reasons for deletion. The first, which we call $P C$ support loss hereafter, is when the unique PC-support $a_{j} \in D\left(x_{j}\right)$ for $a_{i}$ has been deleted. The second, which we call PC-witness loss hereafter, is when the unique PCwitness $a_{j} \in D\left(x_{j}\right)$ for the pair $\left(a_{i}, a_{k}\right)$, where $a_{k}$ is the unique PC-support for $a_{i}$ on some variable $x_{k}$, has been deleted. In both cases value $a_{i}$ is no longer maxRPC.

We now give a unified description of algorithms maxRPC 3 and maxRPC $3^{r m}$. Both algorithms utilize data structures LastPC and Last $A C$ which have the following functionalities: For each constraint $c_{i j}$ and each value $a_{i} \in D\left(x_{i}\right), \operatorname{LastP} C_{x_{i}, a_{i}, x_{j}}$ and Last $A C_{x_{i}, a_{i}, x_{j}}$ give (point to) the most recently discovered PC and AC supports of $a_{i}$ in $D\left(x_{j}\right)$ respectively. Initially, all Last $P C$ and Last $A C$ pointers are set to a special value NIL, considered to precede all values in any domain. Algorithm maxRPC3 updates the LastPC and Last $A C$ structures incrementally like maxRPC2 and AC2001/3.1 respectively do. In contrast, algorithm maxRPC $3^{r m}$ uses these structures as residues like $\operatorname{maxRPC}{ }^{r m}$ and $\mathrm{AC}^{r m}$ do.

The pseudocode for the unified description of maxRPC 3 and maxRPC $3^{r m}$ is given in Algorithm 1 and Functions 2, 3, 4. We assume the existence of a global Boolean variable RM which determines whether the algorithm presented is instantiated to maxRPC3 or to maxRPC $3^{r m}$. If RM is true, the algorithm used is maxRPC $3^{r m}$. Otherwise, the algorithm is maxRPC3.

Being coarse-grained, Algorithm 1 uses a propagation list $L$ where variables that have their domain filtered are inserted. If the algorithm is used for preprocessing then,

\footnotetext{
${ }^{1}$ maxRPC ${ }^{r m}$ also uses residues in a different context.
} 
during an initialization phase, for each value $a_{i}$ of each variable $x_{i}$ we check if $a_{i}$ is maxRPC. If it is not then it is deleted from $D\left(x_{i}\right)$ and $x_{i}$ is added to $L$. The initialization function is not shown in detail due to limited space. If the algorithm is used during search then $L$ is initialized with the currently assigned variable (line 3 ).

In the main part of Algorithm 1 , when a variable $x_{j}$ is removed from $L$, each variable $x_{i}$ constrained with $x_{j}$ must be made maxRPC. For each value $a_{i} \in D\left(x_{i}\right)$ Algorithm 1. like maxRPC2 and maxRPC ${ }^{r m}$, establishes if $a_{i}$ is maxRPC by checking for PCsupport loss and PC-witness loss at lines 8 and 12 .

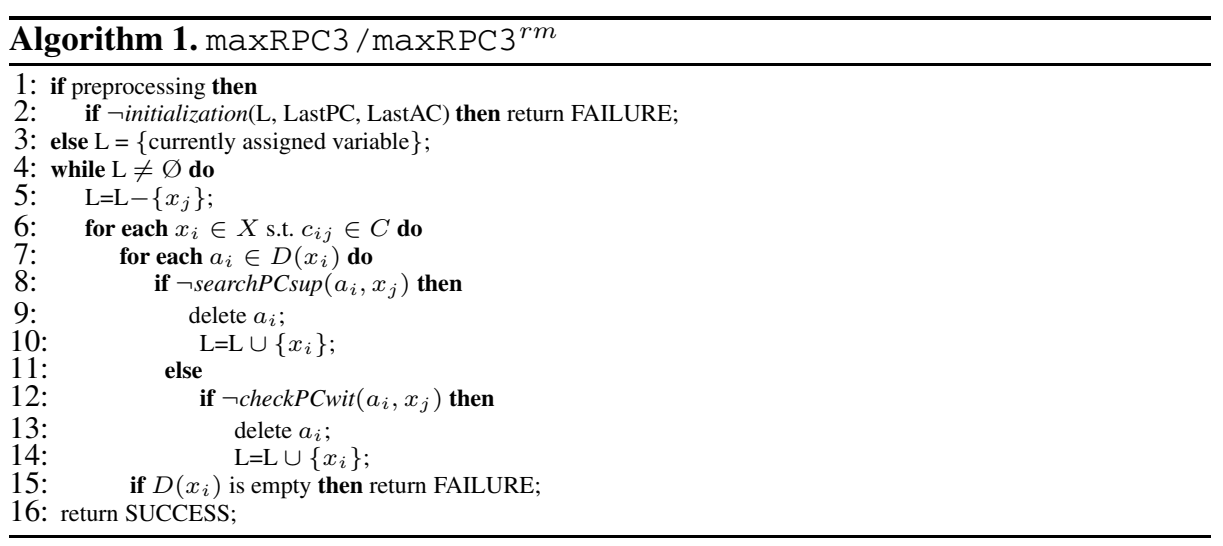

First, function searchPCsup is called to check if a PC-support for $a_{i}$ exists in $D\left(x_{j}\right)$. If value $\operatorname{LastP} C_{x_{i}, a_{i}, x_{j}}$ is still in $D\left(x_{j}\right)$, then searchPCsup returns TRUE (lines 12). If LastPC $C_{x_{i}, a_{i}, x_{j}}$ is not valid, we search for a new PC-support. If maxRPC3 is used, we can take advantage of the LastPC and Last $A C$ pointers to avoid starting this search from scratch. Specifically, we know that no PC-support can exist before LastP $C_{x_{i}, a_{i}, x_{j}}$, and also none can exist before $\operatorname{Last} A C_{x_{i}, a_{i}, x_{j}}$, since all values before Last $A C_{x_{i}, a_{i}, x_{j}}$ are not AC-supports of $a_{i}$. Lines 5-6 in searchPCsup take advantage of these to locate the appropriate starting value $b_{j}$. Note that maxRPC2 always starts the search for a PC-support from the value after LastPC $C_{x_{i}, a_{i}, x_{j}}$. If the algorithm is called during search, in which case we use maxRPC $3^{r m}$ then the search for a new PC-support starts from scratch (line 8), just like maxRPC ${ }^{r m}$ does.

For every value $a_{j} \in D\left(x_{j}\right)$, starting with $b_{j}$, we first check if is an AC-support of $a_{i}$ (line 10). This is done using function isConsistent which simple checks if two values are compatible. If it is, and the algorithm is maxRPC3, then we can update $\operatorname{Last}_{A} C_{x_{i}, a_{i}, x_{j}}$ under a certain condition (lines 12-13). Specifically, if Last $A C_{x_{i}, a_{i}, x_{j}}$ was deleted from $D\left(x_{j}\right)$, then we can set Last $A C_{x_{i}, a_{i}, x_{j}}$ to $a_{j}$ in case $\operatorname{Last} A C_{x_{i}, a_{i}, x_{j}}>$ LastPC $C_{x_{i}, a_{i}, x_{j}}$. If Last $A C_{x_{i}, a_{i}, x_{j}} \leq$ LastPC $C_{x_{i}, a_{i}, x_{j}}$ then we cannot do this as there may be AC-supports for $a_{i}$ between $\operatorname{Last} A C_{x_{i}, a_{i}, x_{j}}$ and $\operatorname{LastPC}_{x_{i}, a_{i}, x_{j}}$ in the lexicographical ordering. We then move on to verify the path consistency of $\left(a_{i}, a_{j}\right)$ through function searchPCwit. 


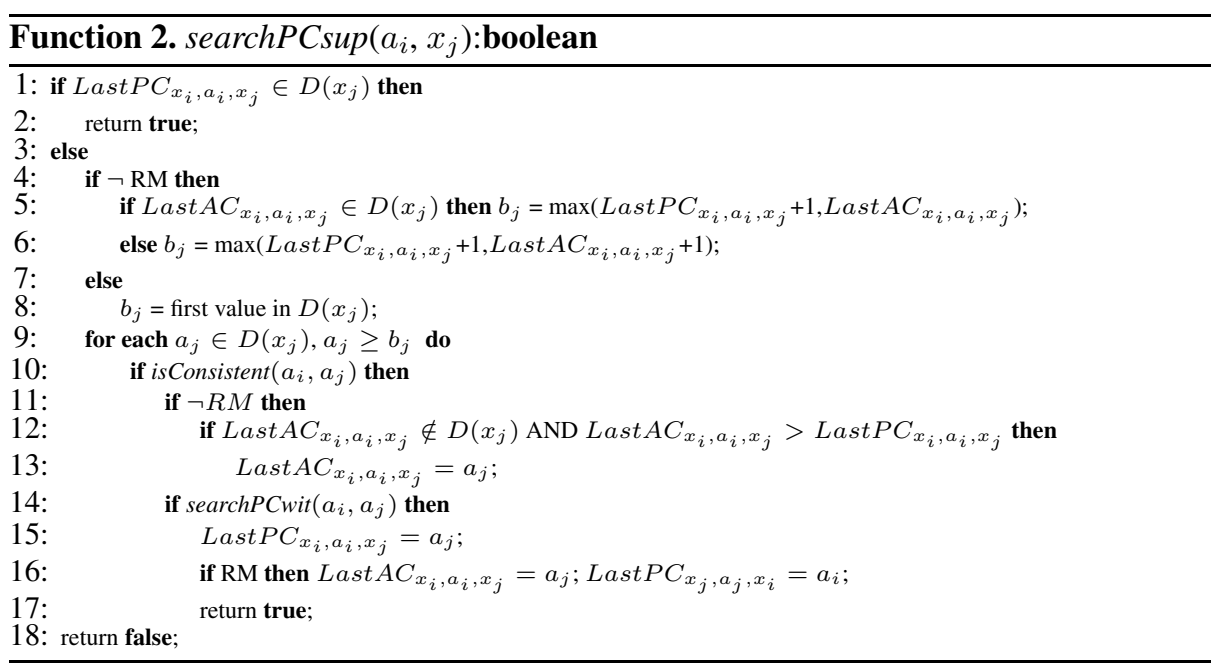

If no PC-support for $a_{i}$ is found in $D\left(x_{j}\right)$, searchPCsup will return FALSE, $a_{i}$ will be deleted and $x_{i}$ will be added to $L$. Otherwise, Last $P C_{x_{i}, a_{i}, x_{j}}$ is set to the discovered PC-support $a_{j}$ (line 15). If maxRPC $3^{r m}$ is used then we update the residue Last $A C_{x_{i}, a_{i}, x_{j}}$ since the discovered PC-support is also an AC-support. In addition, to exploit the multidirectionality of residues, maxRPC $3^{r m}$ sets $\operatorname{LastPC}_{x_{j}, a_{j}, x_{i}}$ to $a_{i}$, as in [10].

Function searchPCwit checks if a pair of values $\left(a_{i}, a_{i}\right)$ is PC by doing the following for each variable $x_{k}$ constrained with $x_{i}$ and $x_{j}$. First, it checks if either Last $A C_{x_{i}, a_{i}, x_{k}}$ is valid and consistent with $a_{j}$ or Last $A C_{x_{j}, a_{j}, x_{k}}$ is valid and consistent with $a_{i}$ (line 3 ). If one of these conditions holds then we have found a PC-witness for $\left(a_{i}, a_{j}\right)$ without searching in $D\left(x_{k}\right)$ and we move on to the next variable constrained with $x_{i}$ and $x_{j}$. Note that neither maxRPC2 nor maxRPC ${ }^{r m}$ can do this as they do not have the Last AC structure. Experimental results in Section 5 demonstrate that these simple conditions can eliminate a very large number of redundant constraint checks.

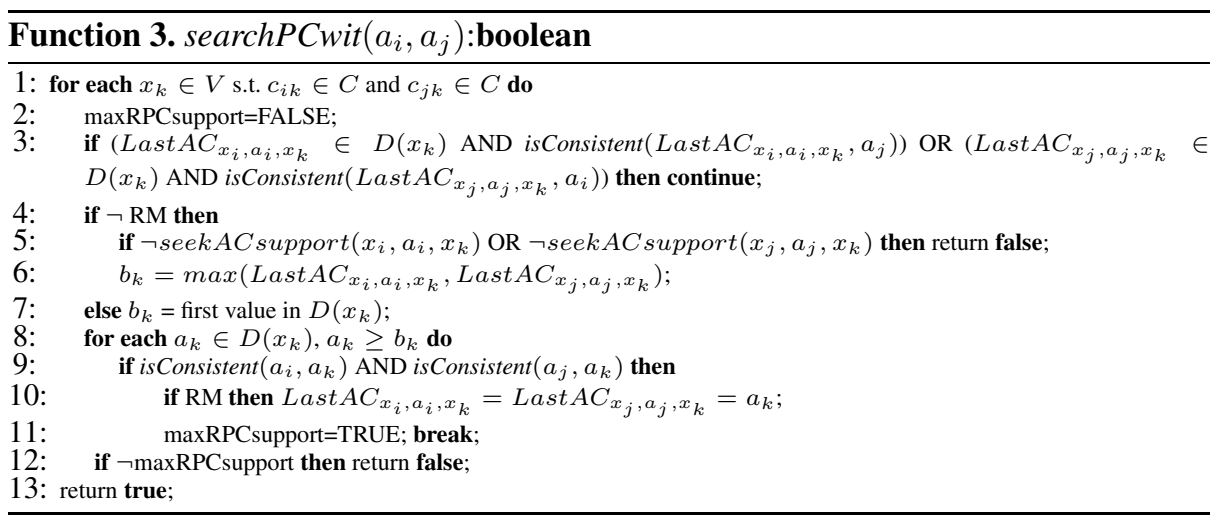

${ }^{2}$ Since AC is enforced by the maxRPC algorithm, we only need to consider variables that form a 3-clique with $x_{i}$ and $x_{j}$. 
If none of the conditions holds then we have to search in $D\left(x_{k}\right)$ for a PC-witness. If the algorithm is maxRPC 3 then we can exploit the Last $A C$ structure to start this search from $b_{k}=\max \left\{\right.$ Last $A C_{x_{i}, a_{i}, x_{k}}$, Last $\left.A C_{x_{j}, a_{j}, x_{k}}\right\}$ (line 6). But before doing this, we call function seekACsupport (not shown for space reasons), first with $\left(x_{i}, a_{i}, x_{k}\right)$ and then with $\left(x_{j}, a_{j}, x_{k}\right)$ as parameters, to find the lexicographically smallest ACsupports for $a_{i}$ and $a_{j}$ in $D\left(x_{k}\right)$ (line 5). If such supports are found, Last $A C_{x_{i}, a_{i}, x_{k}}$ and Last $A C_{x_{j}, a_{j}, x_{k}}$ are updated accordingly. In case no AC-support is found for either $a_{i}$ or $a_{j}$ then seekACsupport returns FALSE, and subsequently searchPCwit() will also return FALSE.

If the algorithm used is maxRPC $3^{r m}$ then we start search for a PC-witness from scratch (line 7), as maxRPC2 and maxRPC ${ }^{r m}$ always do. If a PC-witness $a_{k}$ is found (line 9) and we are using maxRPC $3^{r m}$ then both residues $\operatorname{Last} A C_{x_{i}, a_{i}, x_{k}}$ and Last $A C_{x_{j}, a_{j}, x_{k}}$ are set to $a_{k}$ as they are the most recently discovered AC-supports. If no PC-witness is found then we have determined that the pair $\left(a_{i}, a_{j}\right)$ is not PC and as a result FALSE will be returned and searchPCsup will move to check if the next available value in $D\left(x_{j}\right)$ is a PC-support for $a_{i}$.

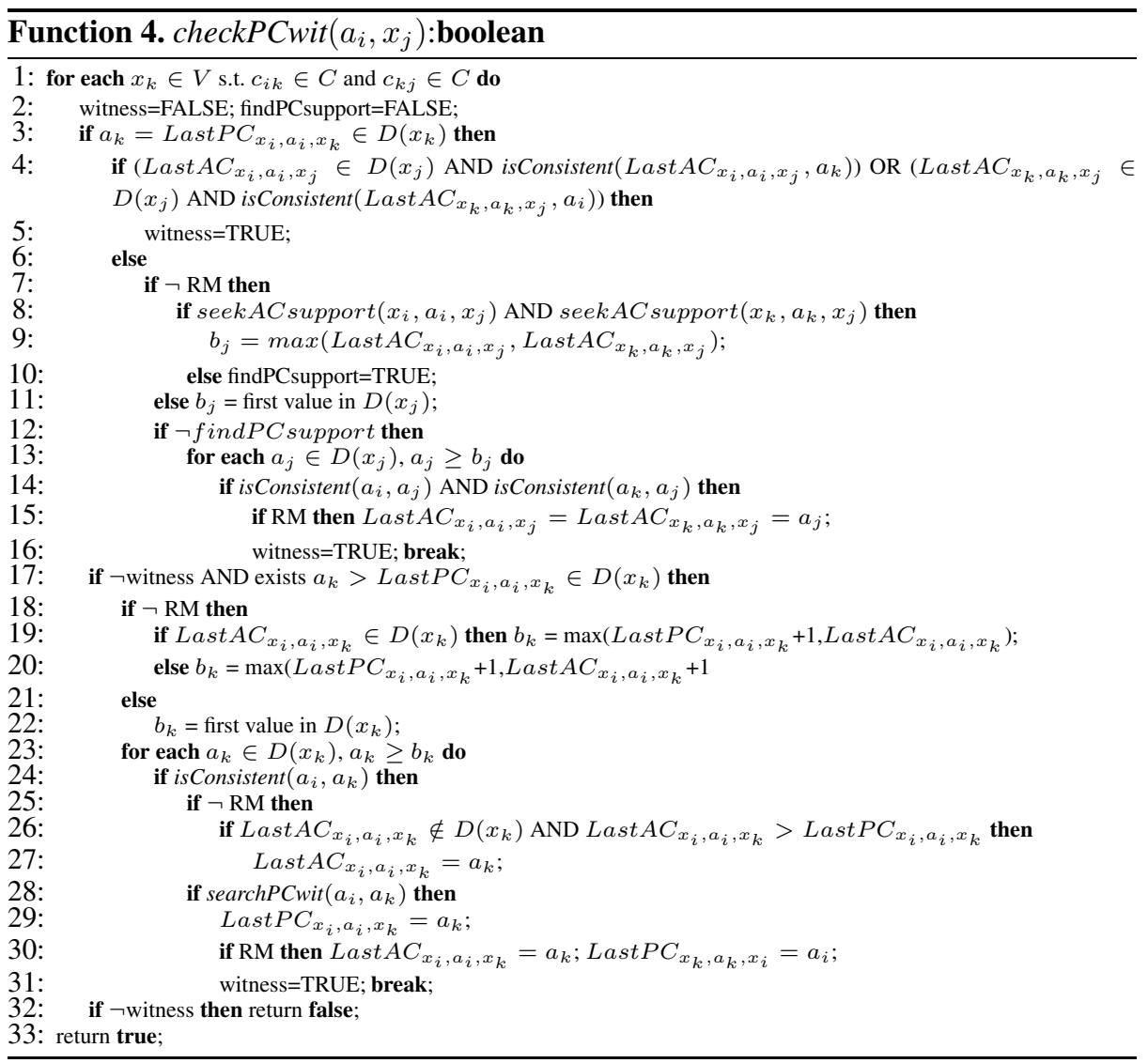


If value $a_{i}$ is not removed by searchPCsup in Algorithm 1, function checkPCwit is called to check for PC-witness loss. This is done by iterating over the variables that are constrained with both $x_{i}$ and $x_{j}$. For each such variable $x_{k}$, we first check if $a_{k}=\operatorname{LastP} C_{x_{i}, a_{i}, x_{k}}$ is still in $D\left(x_{k}\right)$ (line 3 ). If so then we check if there still is a PC-witness in $D\left(x_{j}\right)$. This is done by first checking if either $\operatorname{Last}_{A} C_{x_{i}, a_{i}, x_{j}}$ is valid and consistent with $a_{k}$ or Last $A C_{x_{k}, a_{k}, x_{j}}$ is valid and consistent with $a_{i}$ (line 4). If neither of these conditions holds then we search for a PC-witness starting from $b_{j}=\max \left\{\right.$ Last $A C_{x_{i}, a_{i}, x_{j}}$, Last $\left.A C_{x_{k}, a_{k}, x_{j}}\right\}$ in case of maxRPC3 (line 9), after checking the existence of AC-supports for $a_{i}$ and $a_{k}$ in $D\left(x_{j}\right)$, by calling seekACsupport (line 8). If there is no AC-support in $D\left(x_{j}\right)$ for either $a_{i}$ or $a_{k}$ we set the auxiliary Boolean variable findPCsupport to TRUE to avoid searching for a PC-witness.

If maxRPC $3^{r m}$ is used, we start searching for a PC-witness from scratch (line 11). Note that maxRPC2 does not do the check of line 4 and always starts the search for a PC-witness from the first value in $D\left(x_{j}\right)$. In contrast, maxRPC ${ }^{r m}$ avoids some redundant checks through the use of special residues, albeit resulting in $\mathrm{O}($ end $)$ space complexity. When using maxRPC $3^{r m}$, for each value $a_{j} \in D\left(x_{j}\right)$ we check if it is compatible with $a_{i}$ and $a_{k}$ and move the Last $A C$ pointers accordingly (lines 14-15), exploiting the multidirectionality of residues,

If LastPC $C_{x_{i}, a_{i}, x_{k}}$ has been removed or $a_{i}$ has no PC-witness in $D\left(x_{j}\right)$, we search for a new PC-support for $a_{i}$ in $D\left(x_{k}\right)$. As in function searchPCsup, when maxRPC3 is used this search starts at an appropriate value calculated taking advantage of LastP $C_{x_{i}, a_{i}, x_{k}}$ and Last $A C_{x_{i}, a_{i}, x_{k}}$ (lines 18-20). When maxRPC3 ${ }^{r m}$ is used we start from scratch. If an AC-support for $a_{i}$ is found (line 24), we check if it is also a PC-support by calling function searchPCwit (line 28). If maxRPC3 is used then Last $A C_{x_{i}, a_{i}, x_{k}}$ is updated when necessary (lines 26-27). If a PC-support is found, LastP $C_{x_{i}, a_{i}, x_{k}}$ is set accordingly (line 29). If maxRPC $3^{r m}$ is used then the residue Last $A C_{x_{i}, a_{i}, x_{k}}$ is also updated, as is LastPC $C_{x_{k}, a_{k}, x_{i}}$ (bidirectionality). If the search for a PC-support fails then FALSE will be returned, $a_{i}$ will be deleted, and $x_{i}$ will be added to $\mathrm{L}$.

\subsection{Light maxRPC}

Light maxRPC (lmaxRPC) is an approximation of maxRPC that only propagates the loss of AC-supports and not the loss of PC-witnesses [10]. This ensures that the obtained algorithm enforces a consistency property that is at least as strong as AC.

lmaxRPC is a procedurally defined local consistency, meaning that its description is tied to a specific maxRPC algorithm. Light versions of algorithms maxRPC3 and maxRPC $3^{r m}$, simply noted ImaxRPC 3 and 1 maxRPC $3^{r m}$ respectively, can be obtained by omitting the call to the checkPCwit function (lines 11-14 of Algorithm 1). In a similar way, we can obtain light versions of algorithms maxRPC2 and maxRPC ${ }^{r m}$.

As already noted in [10], the light versions of different maxRPC algorithms may not be equivalent in terms of the pruning they achieve. To give an example, a brute force algorithm for lmaxRPC that does not use any data structures can achieve more pruning than algorithms 1 maxRPC 2,1 maxRPC 3 , and 1 maxRPC ${ }^{r m}$, albeit being much slower in practice. Consider that any of these three algorithms will return TRUE in case LastP $C_{x_{i}, a_{i}, x_{j}}$ is valid. However, although $\operatorname{LastP} C_{x_{i}, a_{i}, x_{j}}$ is valid, it may no longer 
be a PC-support because the PC-witness in some third variable may have been deleted, and it may be the last one. In a case where LastPC $C_{x_{i}, a_{i}, x_{j}}$ was the last PC-support in $x_{j}$ for value $a_{i}$, the three advanced algorithms will not delete $a_{i}$ while the brute force one will. This is because it will exhaustively check all values of $x_{j}$ for PC-support, concluding that there is none.

The worst-case time and space complexities of algorithm 1maxRPC2 are the same as maxRPC2. Algorithm 1 maxRPC ${ }^{r m}$ has $\mathrm{O}\left(n^{3} d^{4}\right)$ time and $\mathrm{O}(e d)$ space complexities, which are lower than those of maxRPC ${ }^{r m}$. Experiments with random problems using algorithms 1 maxRPC ${ }^{r m}$ and maxRPC ${ }^{r m}$ showed that the pruning power of lmaxRPC is only slightly weaker than that of maxRPC [10]. At the same time, it can offer significant gains in run times when used during search. These results were also verified by us through a series of experiments on various problem classes.

\subsection{Complexities}

We now discuss the complexities of algorithms maxRPC 3 and maxRPC $3^{r m}$ and their light versions. To directly compare with existing algorithms for (l)maxRPC, the time complexities give the asymptotic number of constraint checks 3 . Folllowing [9], the node time (resp. space) complexity of a (1)maxRPC algorithm is the worst-case time (resp. space) complexity of invoking the algorithm after a variable assignment. The corresponding branch complexities of an (1)maxRPC algorithm are the worst-case complexities of any incremental sequence of $k \leq n$ invocations of the algorithm. That is, the complexities of incrementally running the algorithm down a branch of the search tree until a fail occurs.

Proposition 1. The node and branch time complexity of (1) maxRPC3 is $\mathrm{O}\left(e n d^{3}\right)$.

Proof. The complexity is determined by the total number of calls to function isConsistent in searchPCsup, checkPCwit, and mainly searchPCwit where most checks are executed.

Each variable can be inserted and extracted from $L$ every time a value is deleted from its domain, giving $\mathrm{O}(d)$ times in the worst case. Each time a variable $x_{j}$ is extracted from $L$, searchPCsup will look for a PC-support in $D\left(x_{j}\right)$ for all values $a_{i} \in D\left(x_{i}\right)$, s.t. $c_{i j} \in C$. For each variable $x_{i}, \mathrm{O}(d)$ values are checked. Checking if a value $a_{j} \in D\left(x_{j}\right)$ is a PC-support involves first checking in $\mathrm{O}(1)$ if it is an AC-support (line 10 in Function 2) and then calling searchPCwit. The cost of searchPCwit is $\mathrm{O}(n+n d)$ since there are $\mathrm{O}(n)$ variables constrained with both $x_{i}$ and $x_{j}$ and, after making the checks in line 3 , their domains must be searched for a PC-witness, each time from scratch with cost $\mathrm{O}(n d)$. Through the use of LastPC no value of $x_{j}$ will be checked more than once over all the $\mathrm{O}(d)$ times $x_{j}$ is extracted from $L$, meaning that for any value $a_{i} \in D\left(x_{i}\right)$ and any variable $x_{j}$, the overall cost of searchPCwit will be $\mathrm{O}\left(d n+n d^{2}\right)=\mathrm{O}\left(n d^{2}\right)$. Hence, searchPCsup will cost $\mathrm{O}\left(n d^{2}\right)$ for one value of $x_{i}$, giving $\mathrm{O}\left(n d^{3}\right)$ for $d$ values. Since, in the worst case, this process will be repeated for every pair of variables $x_{i}$ and $x_{j}$ that are constrained, the total cost of searchPCsup will be $\mathrm{O}\left(e n d^{3}\right)$. This is the node complexity of 1 maxRPC3.

\footnotetext{
${ }^{3}$ However, constraint checks do not always reflect run times as other operations may have an equal or even greater effect.
} 
In checkPCwit the algorithms iterate over the variables in a triangle with $x_{j}$ and $x_{i}$. In the worst case, for each such variable $x_{k}, D\left(x_{j}\right)$ will be searched from scratch for a PC-witness of $a_{i}$ and its current PC-support in $x_{k}$. As $x_{j}$ can be extracted from $L$ $\mathrm{O}(d)$ times and each search from scratch costs $\mathrm{O}(d)$, the total cost of checking for a PC-witness in $D\left(x_{j}\right)$, including the checks of line 4 in Function 4 will be $\mathrm{O}\left(d+d^{2}\right)$. For $d$ values of $x_{i}$ this will be $\mathrm{O}\left(d^{3}\right)$. As this process will be repeated for all triangles of variables, whose number is bounded by $e n$, its total cost will be $\mathrm{O}\left(e n d^{3}\right)$. If no PCwitness is found then a new PC-support for $a_{i}$ in $D\left(x_{k}\right)$ is seeked through searchPCwit. This costs $\mathrm{O}\left(n d^{2}\right)$ as explained above but it is amortized with the cost incurred by the calls to searchPCwit from searchPCsup. Therefore, the cost of checkPCwit is $\mathrm{O}\left(e n d^{3}\right)$. This is also the node complexity of maxRPC3.

The branch complexity of ( 1 ) maxRPC3 is also $\mathrm{O}\left(e n d^{3}\right)$. This is because the use of LastPC ensures that for any constraint $c_{i j}$ and a value $a_{i} \in D\left(x_{i}\right)$, each value of $x_{j}$ will be checked at most once for PC-support while going down the branch. Therefore, the cost of searchPCwit is amortized.

Proposition 2. The node and branch time complexities of 1 maxRPC $3^{r m}$ and maxRPC $3^{r m}$ are $\mathrm{O}\left(e n d^{4}\right)$ and $\mathrm{O}\left(e n^{2} d^{4}\right)$ respectively.

Proof. The proof is not given in detail due to lack of space. The main difference with ImaxRPC 3 is that since lastPC is not updated incrementally, each time we seek a PCsupport for a value $a_{i} \in D\left(x_{i}\right)$ in $x_{j}, D\left(x_{j}\right)$ will be searched from scratch in the worst case. This incurs an extra $\mathrm{O}(d)$ cost to searchPCsup and searchPCwit. Hence, the node complexity of 1 maxRPC $3^{r m}$ is $\mathrm{O}\left(e n d^{4}\right)$. Also, the total cost of searchPCwit in one node cannot be amortized. This means that the cost of searchPCwit within checkPCwit is $\mathrm{O}\left(n d^{2}\right)$. Hence, the node complexity of maxRPC $3^{r m}$ is $\mathrm{O}\left(e n^{2} d^{4}\right)$. The branch complexities are the same because the calls to searchPCwit are amortized.

The space complexities of the algorithms are determined by the space required for data structures Last $P C$ and Last AC. Since both require $\mathrm{O}(e d)$ space, this is the node space complexity of ( 1 ) maxRPC3 and ( 1 ) maxRPC $3^{r m}$. ( 1 ) maxRPC3 has $\mathrm{O}($ end $)$ branch space complexity because of the extra space required for the incremental update and restoration of the data structures. As (1) maxRPC $3^{r m}$ avoid this, its branch space complexity is $\mathrm{O}(e d)$.

\section{Heuristics for maxRPC Algorithms}

Numerous heuristics for ordering constraint or variable revisions have been proposed and used within AC algorithms [113]1]. Heuristics such as the ones used by AC algorithms can be also used within a maxRPC algorithm to efficiently select the next variable to be removed from the propagation list (line 5 of Algorithm 1). In addition to this, maxRPC and lmaxRPC algorithms can benefit from the use of heuristics elsewhere in their execution. Once a variable $x_{j}$ has been removed from the propagation list, heuristics can be applied as follows in either a maxRPC or a lmaxRPC algorithm (we use algorithm ( 1 ) maxRPC3 for illustration): 
1. After a variable $x_{j}$ is removed from $L$ all neighboring variables $x_{i}$ are revised. lmaxRPC (resp. maxRPC) will detect a failure if the condition of PC-support loss (resp. either PC-support or PC-witness loss) occurs for all values of $x_{i}$. In such situations, the sooner $x_{i}$ is considered and the failure is detected, the more constraint checks will be saved. Hence, the order in which the neighboring variables of $x_{j}$ are considered can be determined using a fail-first type of heuristic.

2. Once an AC-support $a_{j} \in D\left(x_{j}\right)$ has been found for a value $a_{i} \in D\left(x_{i}\right)$, searchPCsup tries to establish if it is a PC-support. If there is no PC-witness for the pair $\left(a_{i}, a_{j}\right)$ in some variable $x_{k}$ then $a_{j}$ is not a PC-support. Therefore, we can again use fail-first heuristics to determine the order in which the variables forming a triangle with $x_{i}$ and $x_{j}$ are considered.

The above cases apply to both lmaxRPC and maxRPC algorithms. In addition, a maxRPC algorithm can employ heuristics as follows:

3. For each value $a_{i} \in D\left(x_{i}\right)$ and each variable $x_{k}$ constrained with both $x_{i}$ and $x_{j}$, Function 4 checks if the pair $\left(a_{i}, a_{k}\right)$ still has a PC-witness in $D\left(x_{j}\right)$. If there is no PC-witness or LastPC $C_{x_{i}, a_{i}, x_{k}}$ is not valid then a new PC-support in $x_{k}$ is seeked. If none is found then $a_{i}$ will be deleted. Again heuristics can be used to determine the order in which the variables constrained with $x_{i}$ and $x_{j}$ are considered.

4. In Function 4 if $\operatorname{LastP}_{x_{i}, a_{i}, x_{k}}$ is not valid then a new PC-support for $a_{i}$ in $D\left(x_{k}\right)$ is seeked. The order in which variables constrained with both $x_{i}$ and $x_{k}$ are considered can be determined heuristically as in Case 2 above.

As explained, the purpose of such ordering heuristic will be to "fail-first". That is, to quickly discover potential failures (Case 1 above), refute values that are not PC-supports (Cases 2 and 4) and delete values that have no PC-support (Case 3). Such heuristics can be applied in any coarse-grained maxRPC algorithm to decide the order in which variables are considered in Cases 1-4. Examples are the following:

dom. Consider the variables in ascending domain size. This heuristic can be applied in any of the four cases.

del_ratio. Consider the variables in ascending ratio of the number of remaining values to the initial domain size. This heuristic can be applied in any of the four cases.

wdeg. In Case 1 consider the variables $x_{i}$ in descending weight for the constraint $c_{i j}$. In Case 2 consider the variables $x_{k}$ in descending average weight for the constraints $c_{i k}$ and $c_{j k}$. Similarly for Cases 3 and 4 .

dom/wdeg. Consider the variables in ascending value of dom/wdeg. This heuristic can be applied in any of the four cases.

Experiments demonstrated that applying heuristics in Cases 1 and 3 are particularly effective, while doing so in Cases 2 and 4 saves constraint checks but only marginally reduces cpu times. All of the heuristics mentioned above for Cases 1 and 3 offer cpu gains, with dom/wdeg being the most efficient. Although the primal purpose of the heuristics is to save constraint checks, it is interesting to note that some of the heuristics can also divert search to different areas of the search space when a variable ordering 
heuristic like dom/wdeg is used, resulting in fewer node visits. For example, two different orderings of the variables in Case 1 may result in different constraints causing a failure. As dom/wdeg increases the weight of a constraint each time it causes a failure and uses the weights to select the next variable, this may later result in different branching choices. This is explained for the case of AC in [1].

\section{Experiments}

We have experimented with several classes of structured and random binary CSPs taken from C.Lecoutre's XCSP repository. Excluding instances that were very hard for all algorithms, our evaluation was done on 200 instances in total from various problem classes. More details about these instances can be found in C.Lecoutre's homepage. All algorithms used the dom/wdeg heuristic for variable ordering [4] and lexicographic value ordering. In case of a failure (domain wipe-out) the weight of constraint $c_{i j}$ is updated (right before returning in line 15 of Algorithm 1 ). The suffix ' $+\mathrm{H}$ ' after any algorithm's name means that we have applied the dom/wdeg heuristic for ordering the propagation list [1], and the same heuristic for Case 1 described in Section 4. In absense of the suffix, the propagation list was implemented as a FIFO queue and no heuristic from Section 4 was used.

Table 1 compares the performance of stand-alone algorithms used for preprocessing. We give average results for all the instances, grouped into specific problem classes. We include results from the two optimal coarse-grained maxRPC algorithms, maxRPC2 and maxRPC3, from all the light versions of the coarse-grained algorithms, and from one of the most competitive algorithms (maxRPC3) in tandem with the dom/wdeg heuristics of Section $4(1 \operatorname{maxRPC} 3+\mathrm{H})$. Results show that in terms of run time our algorithms have similar performance and are superior to existing ones by a factor of two on average. This is due to the elimination of many redundant constraint checks as the cc numbers show. Heuristic do not seem to make any difference.

Tables 2 and 3 compare the performance of search algorithms that apply lmaxRPC throughout search on RLFAPs and an indicative collection of other problems respectively. The algorithms compared are $1 \mathrm{maxRPC}{ }^{r m}$ and 1 maxRPC ${ }^{r m}$ with and without

Table 1. Average stand-alone performance in all 200 instances grouped by problem class. Cpu times (t) in secs and constraint checks (cc) are given.

\begin{tabular}{|l|c|c|c|c|c|c|c|c|}
\hline Problem class & & maxRPC2 & maxRPC3 & lmaxRPC2 & lmaxRPC3 & lmaxRPC & lmaxRPC3 $^{r m}$ & $\operatorname{lmaxRPC3+H}$ \\
\hline RLFAP & $\mathrm{t}$ & 6.786 & 2.329 & 4.838 & $\mathbf{2 . 0 4 3}$ & 4.615 & 2.058 & 2.148 \\
(scen,graph) & $\mathrm{cc}$ & $31 \mathrm{M}$ & $9 \mathrm{M}$ & $21 \mathrm{M}$ & $8 \mathrm{M}$ & $21 \mathrm{M}$ & $9 \mathrm{M}$ & $8 \mathrm{M}$ \\
\hline Random & $\mathrm{t}$ & 0.092 & 0.053 & 0.079 & 0.054 & 0.078 & $\mathbf{0 . 0 5 2}$ & 0.056 \\
(modelB,forced) & $\mathrm{cc}$ & $0.43 \mathrm{M}$ & $0.18 \mathrm{M}$ & $0.43 \mathrm{M}$ & $0.18 \mathrm{M}$ & $0.43 \mathrm{M}$ & $0.18 \mathrm{M}$ & $0.18 \mathrm{M}$ \\
\hline Geometric & $\mathrm{t}$ & 0.120 & 0.71 & 0.119 & 0.085 & 0.120 & 0.086 & $\mathbf{0 . 0 7 8}$ \\
& $\mathrm{cc}$ & $0.74 \mathrm{M}$ & $0.35 \mathrm{M}$ & $0.74 \mathrm{M}$ & $0.35 \mathrm{M}$ & $0.74 \mathrm{M}$ & $0.35 \mathrm{M}$ & $0.35 \mathrm{M}$ \\
\hline Quasigroup & $\mathrm{t}$ & 0.293 & 0.188 & 0.234 & 0.166 & 0.224 & $\mathbf{0 . 1 6 1}$ & 0.184 \\
(qcp,qwh,bqwh) & $\mathrm{cc}$ & $1.62 \mathrm{M}$ & $0.59 \mathrm{M}$ & $1.28 \mathrm{M}$ & $0.54 \mathrm{M}$ & $1.26 \mathrm{M}$ & $0.54 \mathrm{M}$ & $0.54 \mathrm{M}$ \\
\hline QueensKnights, & $\mathrm{t}$ & 87.839 & 47.091 & 91.777 & 45.130 & 87.304 & 43.736 & $\mathbf{4 3 . 1 2 1}$ \\
Queens, QueenAttack & $\mathrm{cc}$ & $489 \mathrm{M}$ & $188 \mathrm{M}$ & $487 \mathrm{M}$ & $188 \mathrm{M}$ & $487 \mathrm{M}$ & $188 \mathrm{M}$ & $188 \mathrm{M}$ \\
\hline driver,blackHole & $\mathrm{t}$ & 0.700 & 0.326 & 0.630 & $\mathbf{0 . 2 9 5}$ & 0.638 & 0.303 & 0.299 \\
haystacks,job-shop & $\mathrm{cc}$ & $4.57 \mathrm{M}$ & $1.07 \mathrm{M}$ & $4.15 \mathrm{M}$ & $1.00 \mathrm{M}$ & $4.15 \mathrm{M}$ & $1.00 \mathrm{M}$ & $1.00 \mathrm{M}$ \\
\hline
\end{tabular}


Table 2. Cpu times (t) in secs, nodes (n) and constraint checks (cc) from RLFAP instances. Algorithms that use heuristics are denoted by their name $+\mathrm{H}$. The best cpu time among the lmaxRPC methods is highlighted.

\begin{tabular}{|c|c|c|c|c|c|c|}
\hline instance & & $\mathrm{AC}^{r m}$ & $\operatorname{lmaxRPC}^{r m}$ & $\operatorname{lmaxRPC}^{1 / m}$ & $\operatorname{lmaxRPC}^{r m}+\mathrm{H}$ & $\operatorname{lmaxRPC} 3^{r m}+\mathrm{H}$ \\
\hline \multirow{3}{*}{ scen11 } & $\mathrm{t}$ & 5.4 & 13.2 & 4.6 & \begin{tabular}{|c|}
12.5 \\
\end{tabular} & \begin{tabular}{|c|}
4.3 \\
\end{tabular} \\
\hline & $\mathrm{n}$ & 4,367 & 1,396 & 1,396 & 1,292 & 1,292 \\
\hline & $\mathrm{cc}$ & $5 \mathrm{M}$ & $92 \mathrm{M}$ & $29 \mathrm{M}$ & $90 \mathrm{M}$ & $26 \mathrm{M}$ \\
\hline \multirow[t]{3}{*}{ scen11-f10 } & $\mathrm{t}$ & 11.0 & 29.0 & 12.3 & 22.3 & 9.8 \\
\hline & $\mathrm{n}$ & 9,597 & 2,276 & 2,276 & 1,983 & 1,983 \\
\hline & $\mathrm{cc}$ & $11 \mathrm{M}$ & $141 \mathrm{M}$ & $51 \mathrm{M}$ & $114 \mathrm{M}$ & $41 \mathrm{M}$ \\
\hline \multirow[t]{3}{*}{ scen2-f25 } & $\mathrm{t}$ & 27.1 & 109.2 & 43.0 & 79.6 & 32.6 \\
\hline & $\mathrm{n}$ & 43,536 & 8,310 & 8,310 & 6,179 & 6,179 \\
\hline & $\mathrm{cc}$ & $44 \mathrm{M}$ & $427 \mathrm{M}$ & $151 \mathrm{M}$ & $315 \mathrm{M}$ & $113 \mathrm{M}$ \\
\hline \multirow[t]{3}{*}{ scen3-f11 } & $\mathrm{t}$ & 7.4 & 30.8 & 12.6 & 17.3 & 7.8 \\
\hline & $\mathrm{n}$ & 7,962 & 2,309 & 2,309 & 1,852 & 1,852 \\
\hline & $\mathrm{cc}$ & $9 \mathrm{M}$ & $132 \mathrm{M}$ & $46 \mathrm{M}$ & $80 \mathrm{M}$ & $29 \mathrm{M}$ \\
\hline \multirow[t]{3}{*}{ scen11-f7 } & $\mathrm{t}$ & $4,606.5$ & $8,307.5$ & $3,062.8$ & $6,269.0$ & $2,377.6$ \\
\hline & $\mathrm{n}$ & $3,696,154$ & 552,907 & 552,907 & 522,061 & 522,061 \\
\hline & $\mathrm{cc}$ & $4,287 \mathrm{M}$ & $35,897 \mathrm{M}$ & $9,675 \mathrm{M}$ & $22,899 \mathrm{M}$ & $6,913 \mathrm{M}$ \\
\hline \multirow[t]{3}{*}{ scen11-f8 } & $\mathrm{t}$ & 521.1 & $2,680.6$ & 878.0 & $1,902.4$ & 684.7 \\
\hline & $\mathrm{n}$ & 345,877 & 112,719 & 112,719 & 106,352 & 106,352 \\
\hline & $\mathrm{cc}$ & $638 \mathrm{M}$ & $10,163 \mathrm{M}$ & $3,172 \mathrm{M}$ & $7,585 \mathrm{M}$ & $2,314 \mathrm{M}$ \\
\hline \multirow[t]{3}{*}{ graph8-f10 } & $\mathrm{t}$ & 16.4 & 16.8 & 9.1 & 11.0 & 6.3 \\
\hline & $\mathrm{n}$ & 18,751 & 4,887 & 4,887 & 3,608 & 3,608 \\
\hline & $\mathrm{cc}$ & $14 \mathrm{M}$ & $71 \mathrm{M}$ & $31 \mathrm{M}$ & $51 \mathrm{M}$ & $21 \mathrm{M}$ \\
\hline \multirow[t]{3}{*}{ graph14-f28 } & $\mathrm{t}$ & 31.4 & 4.1 & 3.1 & 2.6 & 2.1 \\
\hline & $\mathrm{n}$ & 57,039 & 2,917 & 2,917 & 1,187 & 1,187 \\
\hline & $\mathrm{cc}$ & $13 \mathrm{M}$ & $17 \mathrm{M}$ & $8 \mathrm{M}$ & $13 \mathrm{M}$ & $6 \mathrm{M}$ \\
\hline \multirow[t]{3}{*}{ graph9-f9 } & $\mathrm{t}$ & 273.5 & 206.3 & 101.5 & 289.5 & 146.9 \\
\hline & $\mathrm{n}$ & 273,766 & 26,276 & 26,276 & 49,627 & 49,627 \\
\hline & $\mathrm{cc}$ & $158 \mathrm{M}$ & $729 \mathrm{M}$ & $290 \mathrm{M}$ & $959 \mathrm{M}$ & $371 \mathrm{M}$ \\
\hline
\end{tabular}

the use of heuristic dom/wdeg for propagation list and for Case 1 of Section 4 We also include results from $\mathrm{MAC}^{r m}$ which is considered the most efficient version of MAC [8].

Experiments showed that 1 maxRPC ${ }^{r m}$ is the most efficient among existing algorithms when applied during search, which confirms the results given in [10]. Accordingly, lmaxRPC $3^{r m}$ is the most efficient among our algorithms. It is between two and four times faster than maxRPC $3^{r m}$ on hard instances, while algorithms 1maxRPC3 and ImaxRPC2 are not competitive when used during search because of the data structures they maintain. In general, when applied during search, any maxRPC algorithm is clearly inferior to the corresponding light version. The reduction in visited nodes achieved by the former is relatively small and does not compensate for the higher run times of enforcing maxRPC.

Results from Tables 2 and 3 demonstrate that 1 maxRPC $3^{r m}$ always outperforms 1 maxRPC ${ }^{r m}$, often considerably. This was the case in all 200 instances tried. The use of heuristics improves the performance of both lmaxRPC algorithms in most cases. Looking at the columns for 1 maxRPC ${ }^{r m}$ and 1 maxRPC $3^{r m}+\mathrm{H}$ we can see that our methods can reduce the numbers of constraint checks by as much as one order of magnitude (e.g. in quasigroup problems qcp and qwh). This is mainly due to the elimination of redundant checks inside function searchPCwit. Cpu times are not cut down by as much, but a speed-up of more than 3 times can be obtained (e.g. scen2-f25 and scen11-f8).

Importantly, the speed-ups obtained can make a search algorithm that efficiently applies lmaxRPC competitive with MAC on many instances. For instance, in scen11-f10 
Table 3. Cpu times (t) in secs, nodes (n) and constraint checks (cc) from various instances

\begin{tabular}{|c|c|c|c|c|c|c|}
\hline instance & & $\mathrm{AC}^{r m}$ & $\operatorname{lmaxRPC}^{r m}$ & $\operatorname{lmaxRPC} 3^{r m}$ & $\operatorname{lmaxRPC}{ }^{r m}+\mathrm{H}$ & $\operatorname{lmaxRPC} 3^{r m}+\mathrm{H}$ \\
\hline rand-2-40-8 & $\mathrm{t}$ & 4.0 & 47.3 & 21.7 & 37.0 & 19.0 \\
\hline \multirow[t]{2}{*}{$-753-100-75$} & $\mathrm{n}$ & 13,166 & 8,584 & 8,584 & 6,915 & 6,915 \\
\hline & $\mathrm{cc}$ & $7 \mathrm{M}$ & $289 \mathrm{M}$ & $82 \mathrm{M}$ & $207 \mathrm{M}$ & $59 \mathrm{M}$ \\
\hline geo50-20 & $\mathrm{t}$ & 102.7 & 347.7 & 177.5 & 273.3 & 150.3 \\
\hline \multirow[t]{2}{*}{ d4-75-1 } & $\mathrm{n}$ & 181,560 & 79,691 & 79,691 & 75,339 & 75,339 \\
\hline & $\mathrm{cc}$ & $191 \mathrm{M}$ & $2,045 \mathrm{M}$ & $880 \mathrm{M}$ & $1,437 \mathrm{M}$ & $609 \mathrm{M}$ \\
\hline \multirow[t]{3}{*}{ qcp150-120-5 } & $\mathrm{t}$ & 52.1 & 89.4 & 50.2 & 80.0 & 55.3 \\
\hline & $\mathrm{n}$ & 233,311 & 100,781 & 100,781 & 84,392 & 84,392 \\
\hline & $\mathrm{cc}$ & $27 \mathrm{M}$ & $329 \mathrm{M}$ & $53 \mathrm{M}$ & $224 \mathrm{M}$ & $36 \mathrm{M}$ \\
\hline \multirow[t]{3}{*}{ qcp150-120-9 } & $\mathrm{t}$ & 226.8 & 410.7 & 238.1 & 239.9 & 164.3 \\
\hline & $\mathrm{n}$ & $1,195,896$ & 583,627 & 583,627 & 315,582 & 315,582 \\
\hline & $\mathrm{cc}$ & $123 \mathrm{M}$ & $1,613 \mathrm{M}$ & $250 \mathrm{M}$ & $718 \mathrm{M}$ & $112 \mathrm{M}$ \\
\hline \multirow[t]{3}{*}{ qwh20-166-1 } & $\mathrm{t}$ & 52.6 & 64.3 & 38.9 & 21.2 & 14.9 \\
\hline & $\mathrm{n}$ & 144,653 & 44,934 & 44,934 & 13,696 & 13,696 \\
\hline & $\mathrm{cc}$ & $19 \mathrm{M}$ & $210 \mathrm{M}$ & $23 \mathrm{M}$ & $53 \mathrm{M}$ & $6 \mathrm{M}$ \\
\hline \multirow[t]{3}{*}{ qwh20-166-6 } & $\mathrm{t}$ & $1,639.0$ & $1,493.5$ & 867.1 & $1,206.2$ & 816.5 \\
\hline & $\mathrm{n}$ & $4,651,632$ & 919,861 & 919,861 & 617,233 & 617,233 \\
\hline & $\mathrm{cc}$ & $633 \mathrm{M}$ & $5,089 \mathrm{M}$ & $566 \mathrm{M}$ & $3,100 \mathrm{M}$ & $351 \mathrm{M}$ \\
\hline \multirow[t]{3}{*}{ qwh20-166-9 } & $\mathrm{t}$ & 41.8 & 41.1 & 25.0 & 39.9 & 28.5 \\
\hline & $\mathrm{n}$ & 121,623 & 32,925 & 32,925 & 26,505 & 26,505 \\
\hline & $\mathrm{cc}$ & $15 \mathrm{M}$ & $135 \mathrm{M}$ & $15 \mathrm{M}$ & $97 \mathrm{M}$ & $11 \mathrm{M}$ \\
\hline \multirow{3}{*}{$\begin{array}{l}\text { blackHole } \\
4-4-e-8\end{array}$} & $\mathrm{t}$ & 1.8 & 14.4 & 3.8 & 12.1 & 3.6 \\
\hline & $\mathrm{n}$ & 8,661 & 4,371 & 4,371 & 4,325 & 4,325 \\
\hline & $\mathrm{cc}$ & $4 \mathrm{M}$ & $83 \mathrm{M}$ & $12 \mathrm{M}$ & $68 \mathrm{M}$ & $10 \mathrm{M}$ \\
\hline \multirow[t]{3}{*}{ queens-100 } & $\mathrm{t}$ & 15.3 & 365.3 & 106.7 & 329.8 & 103.0 \\
\hline & $\mathrm{n}$ & 7,608 & 6,210 & 6,210 & 5,030 & 5,030 \\
\hline & $\mathrm{cc}$ & $6 \mathrm{M}$ & $1,454 \mathrm{M}$ & $377 \mathrm{M}$ & $1,376 \mathrm{M}$ & $375 \mathrm{M}$ \\
\hline \multirow[t]{3}{*}{ queenAttacking5 } & $\mathrm{t}$ & 34.3 & 153.1 & 56.7 & 136.0 & 54.8 \\
\hline & $\mathrm{n}$ & 139,534 & 38,210 & 38,210 & 33,341 & 33,341 \\
\hline & $\mathrm{cc}$ & $35 \mathrm{M}$ & $500 \mathrm{M}$ & $145 \mathrm{M}$ & $436 \mathrm{M}$ & $128 \mathrm{M}$ \\
\hline \multirow{3}{*}{$\begin{array}{l}\text { queensKnights } \\
-15-5-\mathrm{mul}\end{array}$} & $\mathrm{t}$ & 217.0 & 302.0 & 173.6 & 482.0 & 283.5 \\
\hline & $\mathrm{n}$ & 35,445 & 13,462 & 13,462 & 12,560 & 12,560 \\
\hline & $\mathrm{cc}$ & $153 \mathrm{M}$ & $963 \mathrm{M}$ & $387 \mathrm{M}$ & $1,795 \mathrm{M}$ & $869 \mathrm{M}$ \\
\hline
\end{tabular}

Table 4. Average search performance in all 200 instances grouped by class

\begin{tabular}{|l|c|c|c|c|c|c|}
\hline Problem class & & $\mathrm{AC}^{r m}$ & $\operatorname{lmaxRPC}^{r m}$ & $\operatorname{lmaxRPC}^{r m}$ & $\operatorname{lmaxRPC}^{r m}+\mathrm{H}$ & $\operatorname{lmaxRPC}^{r m}+\mathrm{H}$ \\
\hline RLFAP & $\mathrm{t}$ & 242.8 & 556.7 & 199.3 & 416.3 & $\mathbf{1 5 7 . 3}$ \\
(scen,graph) & $\mathrm{cc}$ & $233 \mathrm{M}$ & $2,306 \mathrm{M}$ & $663 \mathrm{M}$ & $1,580 \mathrm{M}$ & $487 \mathrm{M}$ \\
\hline Random & $\mathrm{t}$ & $\mathbf{8 . 4}$ & 28.0 & 14.8 & 28.5 & 17.1 \\
(modelB,forced) & $\mathrm{cc}$ & $14 \mathrm{M}$ & $161 \mathrm{M}$ & $60 \mathrm{M}$ & $137 \mathrm{M}$ & $51 \mathrm{M}$ \\
\hline Geometric & $\mathrm{t}$ & $\mathbf{2 1 . 5}$ & 72.2 & 37.2 & 57.6 & 32.1 \\
& $\mathrm{cc}$ & $39 \mathrm{M}$ & $418 \mathrm{M}$ & $179 \mathrm{M}$ & $297 \mathrm{M}$ & $126 \mathrm{M}$ \\
\hline Quasigroup & $\mathrm{t}$ & 147.0 & 162.5 & 94.9 & 128.9 & $\mathbf{8 9 . 6}$ \\
(qcp,qwh,bqwh) & $\mathrm{cc}$ & $59 \mathrm{M}$ & $562 \mathrm{M}$ & $68 \mathrm{M}$ & $333 \mathrm{M}$ & $40 \mathrm{M}$ \\
\hline QueensKnights, & $\mathrm{t}$ & $\mathbf{9 0 . 2}$ & 505.2 & 180.3 & 496.4 & 198.1 \\
Queens, QueenAttack & $\mathrm{cc}$ & $74 \mathrm{M}$ & $1,865 \mathrm{M}$ & $570 \mathrm{M}$ & $1,891 \mathrm{M}$ & $654 \mathrm{M}$ \\
\hline driver,blackHole & $\mathrm{t}$ & $\mathbf{3 . 2}$ & 17.1 & 9.1 & 11.9 & 7.0 \\
haystacks,job-shop & $\mathrm{cc}$ & $1.8 \mathrm{M}$ & $55 \mathrm{M}$ & $6.4 \mathrm{M}$ & $36.7 \mathrm{M}$ & $5.1 \mathrm{M}$ \\
\hline
\end{tabular}

we achieve the same run time as MAC while 1 maxRPC ${ }^{r m}$ is 3 times slower while in scen11-f7 we go from 2 times slower to 2 times faster. In addition, there are several instances where MAC is outperformed (e.g. the graph RLFAPs and most quasigroup problems). Of course, there are still instances where MAC remains considerably faster despite the improvements.

Table 4 summarizes results from the application of lmaxRPC during search. We give average results for all the tested instances, grouped into specific problem classes. As 
can be seen, our best method improves on the existing best one considerably, making lmaxRPC outperform MAC on the RFLAP and quasigroup problem classes. Overall, our results demonstrate that the efficient application of a maxRPC approximation throughout search can give an algorithm that is quite competitive with MAC on many binary CSPs. This confirms the conjecture of [6] about the potential of maxRPC as an alternative to AC. In addition, our results, along with ones in [10], show that approximating strong and complex local consistencies can be very beneficial.

\section{Conclusion}

We presented maxRPC 3 and maxRPC $3^{r m}$, two new algorithms for maxRPC, and their light versions that approximate maxRPC. These algorithms build on and improve existing maxRPC algorithms, achieving the elimination of many redundant constraint checks. We also investigated heuristics that can be used to order certain operations within maxRPC algorithms. Experimental results from various problem classes demonstrate that our best method, 1 maxRPC $3^{r m}$, constantly outperforms existing algorithms, often by large margins. Significantly, the speed-ups obtained allow lmaxRPC $3^{r m}$ to compete with and outperform MAC on many problems. In the future we plan to adapt techniques for using residues from [9] to improve the performance of our algorithms during search. Also, it would be interesting to investigate the applicability of similar methods to efficiently achieve or approximate other local consistencies.

\section{References}

1. Balafoutis, T., Stergiou, K.: Exploiting constraint weights for revision ordering in Arc Consistency Algorithms. In: ECAI 2008 Workshop on Modeling and Solving Problems with Constraints (2008)

2. Bessière, C., Régin, J.C., Yap, R., Zhang, Y.: An Optimal Coarse-grained Arc Consistency Algorithm. Artificial Intelligence 165(2), 165-185 (2005)

3. Boussemart, F., Hemery, F., Lecoutre, C.: Revision ordering heuristics for the Constraint Satisfaction Problem. In: CP-2004 Workshop on Constraint Propagation (2004)

4. Boussemart, F., Hemery, F., Lecoutre, C., Sais, L.: Boosting systematic search by weighting constraints. In: Proceedings of ECAI-2004 (2004)

5. Debruyne, R., Bessière, C.: From restricted path consistency to max-restricted path consistency. In: Smolka, G. (ed.) CP 1997. LNCS, vol. 1330, pp. 312-326. Springer, Heidelberg (1997)

6. Debruyne, R., Bessière, C.: Domain Filtering Consistencies. JAIR 14, 205-230 (2001)

7. Grandoni, F., Italiano, G.: Improved Algorithms for Max-Restricted Path Consistency. In: Rossi, F. (ed.) CP 2003. LNCS, vol. 2833, pp. 858-862. Springer, Heidelberg (2003)

8. Lecoutre, C., Hemery, F.: A study of residual supports in arc cosistency. In: Proceedings of IJCAI 2007, pp. 125-130 (2007)

9. Likitvivatanavong, C., Zhang, Y., Bowen, J., Shannon, S., Freuder, E.: Arc Consistency during Search. In: Proceedings of IJCAI 2007, pp. 137-142 (2007)

10. Vion, J., Debruyne, R.: Light Algorithms for Maintaining Max-RPC During Search. In: Proceedings of SARA 2009 (2009)

11. Wallace, R., Freuder, E.: Ordering heuristics for arc consistency algorithms. In: AI/GI/VI, Vancouver, British Columbia, Canada, pp. 163-169 (1992) 